\title{
Thermodynamics properties of the dark energy in loop quantum cosmology
}

\author{
Kui Xiad* and Jian-Yang Zhü \\ Department of Physics, Beijing Normal University, Beijing 100875, China
}

(Dated: June 27, 2018)

\begin{abstract}
Considering an arbitrary, varying equation of the state parameter, the thermodynamic properties of the dark energy fluid in a semiclassical loop quantum cosmology scenario, which we consider the inverse volume modification, is studied. The equation of the state parameters are corrected as a semiclassical one during considering the effective behavior. Assuming that the apparent horizon has Hawking temperature, the modified entropy-area relation is obtained, we find that this relation is different from the one which is obtained by considering the holonomy correction. Considering the dark energy is a thermal equilibrium fluid, we get the expressions for modified temperature, chemical potential and entropy. The temperature, chemical potential and entropy are well-defined in the semiclassical regions.
\end{abstract}

PACS numbers: 04.60.Pp, 04.60.Kz, 98.80.Qc

\section{INTRODUCTION}

More and more evidences [1] show that our universe may be dominated by dark energy (for more recent review, please read [2] and references therein.). Although the cosmology constant $\Lambda$ maybe responsible for the observed data, but it is also the possible that there are exiting the dynamical mechanism is at work. There are some candidates to describe those dynamical systems, e.g., a canonical scalar field (quintessence) [3], a scalar field with negative sign of kinetic term (phantom) [4], or the combination of quintessence and phantom in a unified model named quintom [5] and so on [2]. Many authors have analysed the characters of dark energy in the classical cosmology, e.g., the inflation caused by dark energy [6], attractor behavior and solution 7], the relationship between geometry and dark energy [8], the fate of universe with dark energy 9], the singularity of universe with dark energy [10], the thermodynamical properties of dark energy [11] and so on. And some authors studied the quantum properties of it 12]. But we still have very little knowledge about it, and there is not any direct evidence shows whether the dark energy exists. So it is necessary to study the properties of it.

The universe can be considered as a thermodynamical system. The thermodynamical properties of the universe was studied by many authors [13, 14]. The apparent horizon and event horizon of universe are different, the first and second law of thermodynamics hold on the apparent horizon, but they break down while we consider the event horizon [14]. According to [15], the thermodynamical properties of the universe in loop quantum cosmology (LQC) is still held. But the corrected Firedmann equation will modify the entropy-area relation of apparent horizon in LQC [16]. Due to this subtlety, we will focus our attention on the thermodynamical prop-

\footnotetext{
*Electronic address: 87xiaokui@mail.bnu.edu.cn
}

${ }^{\dagger}$ Electronic address: zhujy@bnu.edu.cn erties of the apparent horizon first. And then we will derive thermodynamical variables of dark energy fluid in the inner side of the apparent horizon in the semiclassical LQC scenario.

Loop quantum cosmology (for more recent review, please read [17, 18]) is a canonical quantization of homogeneous spactimes based upon techniques used in loop quantum gravity (LQG). Due to the homogeneous and isotropic spacetime, the phase space of LQC is simpler than LQG. The connection is determined by a single parameter $c$ and the triad is determined by $p$. The variables $c$ and $p$ are canonically conjugate with Poisson bracket $\{c, p\}=\frac{8 \pi G}{3} \gamma$, in which $\gamma$ is the Barbero-Immirzi parameter. In the LQC scenario, the initial singularity is instead by a bounce. So, thanks for the quantum effect, the universe is initially contracting phase with the minimal but not zero volume, and then the quantum effect drives it to the expanding phase. If one wants to consider the physical effect near the minimal area, e.g., very small scale factor $a<a_{i}=\sqrt{\gamma} l_{\mathrm{pl}}$, it is necessary to consider the difference equation 19]. But if we consider the interval $a_{i}<a<a_{\star}=\sqrt{\gamma j / 3} l_{\mathrm{pl}}$, with the quantum parameter $j$, we can consider the effective dynamics of LQC. And the classical cosmology can be recovered if $a>a_{\star}$. In this paper, we just consider the thermodynamical of the dark energy fluid in the semiclassical regions.

In the classical regions, the dark energy thermodynamics has been studied by many authors [11]. The thermodynamics properties of dark energy fluid described by the equation of state parameter $\omega_{\mathrm{cl}}=P_{\mathrm{cl}} / \rho_{\mathrm{cl}}$, in which $P_{\mathrm{cl}}, \rho_{\mathrm{cl}}$ are the pressure and energy density of dark energy fluids respective. For the thermodynamical properties of quintessence filed, with a constant $\omega_{\mathrm{cl}}>-1$, it is nature to use the results of conventional perfect fluid thermodynamics [20]. But, in the case of phantom fields, it is more complex if we consider constant $\omega_{\mathrm{cl}}<-1$. Considering the chemical potential $\mu=0$, some authors showed that the energy density and entropy are positive, but the temperature is negative [21, 22], and they argued that this negative temperature due to the quantum properties of phantom fields. And some other authors 
showed that, if the temperature, energy density and entropy is positive, one must get a negative chemical potential [23]. But considering the varying $\omega_{\mathrm{cl}}(a)$, one can obtain more physics contents, well-defined temperature, energy density, chemical potential, and entropy on the case of $\omega_{\mathrm{cl}}(a)=-1$, but the temperature will divergence when $a=0$ in the classical universe [20]. In addition, if considering that the temperature of dark energy fluids have the same temperature of the Hawking temperature on the apparent horizon, one maybe get negative entropy in condition of $\omega_{\mathrm{cl}}(a)<-1$ [24].

Based on those confused properties of the thermodynamics of dark energy fluids, it is necessary to discuss it in the semiclassical regions, even quantum regions. In this paper, we will focus our interested on the semiclassical one based on the effective LQC. Just as the above discussion, the scale factor should be in the regions of $a_{i}<a<a_{\star}$. The aim of this paper is tripartite. The first is to get the modified equation of state parameters $\omega_{\mathrm{sc}}$ in the semiclassical LQC. The second is to discuss the modified entropy-area relation of apparent horizon which is caused by the corrected Firedmann equation. And the last is to obtain the expressions of thermodynamical variables of dark energy fluids which is in the inner side of the apparent horizon.

The organization of this paper is as follows. In Sec. II we will introduce the basic concepts of semiclassical LQC and get the modified varying $\omega_{\mathrm{sc}}(a)$. And then in Section III, we will obtain the modified entropy-area relation of the apparent horizon and the expressions of thermodynamical variables of the dark energy fluid inside of the apparent horizon. In the last section IV we will get some conclusions and discussions.

\section{MODIFIED EFFECTIVE DYNAMICS IN LQC}

The basic variables of phase space of LQC are the connection $c$ and the triad $p$. In our interested background spacetime, the connection and triad can be related to the scale factor, $c=\gamma \dot{a}, p=a^{2}=V^{2 / 3}$, with the BarberoImmirzi parameter $\gamma \approx 0.2375$. The connection and triad satisfy the Possion bracket

$$
\{c, p\}=\frac{8 \pi G}{3} \gamma
$$

When the scale factor lies in the regions $a_{i}<a<a_{\star}$, we consider the effective dynamics of LQC, with $a_{i}=\sqrt{\gamma} l_{\mathrm{pl}}$, $a_{\star}=\sqrt{\gamma j / 3} l_{\mathrm{pl}}$, and $j$ is the quantum parameter and must be taken half integer values. If $a<a_{i}$, the quantum effect dominates, we must consider the difference equation. And $a>a_{\star}$, we turn to the classical effect. In this paper, we just consider the semiclassical effect of LQC. The effective Hamiltonian can be written as [25, 26]

$$
\mathcal{H}_{\mathrm{eff}}=-\frac{3}{\gamma^{2} 8 \pi G} \mathcal{S} a\left[(c-1)^{2}+\gamma^{2}\right]+\mathcal{H}_{\mathrm{m}},
$$

with the dark energy Hamiltonian $\mathcal{H}_{\mathrm{m}}$. And $\mathcal{S}$ is

$$
\begin{aligned}
\mathcal{S}= & \frac{1}{4}\left[2\left((q+1)^{3}-|q-1|^{3}\right)\right. \\
& \left.-3 q\left((q+1)^{2}-\operatorname{sgn}(\mathrm{q}-1)|\mathrm{q}-1|^{2}\right)\right]
\end{aligned}
$$

with $q=a^{2} / a_{\star}^{2}$.

One can get the equations of motion of connection and triad from the Hamiltonian (2)

$$
\begin{aligned}
\dot{p} & =\left\{p, \mathcal{H}_{\mathrm{eff}}\right\}=-\frac{8 \pi G \gamma}{3} \frac{\partial \mathcal{H}_{\mathrm{eff}}}{\partial c}=\frac{2}{\gamma} \mathcal{S} a(c-1), \\
\dot{c} & =\left\{c, \mathcal{H}_{\mathrm{eff}}\right\}=\frac{8 \pi G \gamma}{3} \frac{\partial \mathcal{H}_{\mathrm{eff}}}{\partial p} \\
& =-\frac{(c-1)^{2}+\gamma^{2}}{2 \gamma}\left(\frac{\mathcal{S}}{a}+\frac{\dot{\mathcal{S}}}{\dot{a}}\right)+\frac{8 \pi G \gamma}{3} \frac{\partial \mathcal{H}_{\mathrm{m}}}{\partial p}
\end{aligned}
$$

where dot denotes the derivation with respect to the time $t$. Considering Eq. (44) and remembering the Hamiltonian constrain satisfies $\mathcal{H}_{\text {eff }} \approx 0$, one can obtain the modified Friedmann equation

$$
H^{2}=\left(\frac{\dot{a}}{a}\right)^{2}=\frac{8 \pi G}{3} \mathcal{S} \rho_{\mathrm{sc}}-\frac{\mathcal{S}^{2}}{a^{2}},
$$

with the Hubble parameter $H=\dot{a} / a$ and the modified dark energy density $\rho_{\mathrm{sc}}=E_{\mathrm{m}} / a^{3} . E_{\mathrm{m}}$ is the eigenvalues for the dark energy Hamiltonian operator that includes the appropriate modifications to the inverse volume [26]. We will discuss the definition of $\rho_{\mathrm{sc}}$ later on.

Considering the modified pressure can be written as $P_{\mathrm{sc}}=-\frac{\partial \mathcal{H}_{\mathrm{m}}}{\partial V}=-\frac{2}{3} p^{-1 / 2} \frac{\partial \mathcal{H}_{\mathrm{m}}}{\partial p}$, and using the equations of motion of connection and triad (4) and (5), one can obtain the modified Raychaudhuri equation

$$
\frac{\ddot{a}}{a}=-\frac{4 \pi G}{3} \mathcal{S}\left(\rho_{\mathrm{sc}}+3 P_{\mathrm{sc}}\right)+\frac{1}{2} \frac{\dot{\mathcal{S}}}{a}\left(\frac{\dot{a}}{\mathcal{S}}-\frac{\mathcal{S}}{\dot{a}}\right) .
$$

Combining the Friedmann equation (6) and the Raychaudhuri equation (7), one can get

$$
\begin{aligned}
\dot{H}= & \frac{\ddot{a}}{a}-\frac{\dot{a}^{2}}{a^{2}}=-\frac{8 \pi G}{2} \mathcal{S}\left(\rho_{\mathrm{sc}}+P_{\mathrm{sc}}\right) \\
& +\frac{1}{2} \frac{\dot{\mathcal{S}}}{a}\left[\frac{\dot{a}}{\mathcal{S}}-\frac{\mathcal{S}}{\dot{a}}\right]+\frac{\mathcal{S}^{2}}{a^{2}}
\end{aligned}
$$

and it is easy to verify the conservation equation

$$
\dot{\rho}_{\mathrm{sc}}+3 H\left(\rho_{\mathrm{sc}}+P_{\mathrm{sc}}\right)=0 .
$$

Defining the equation of state $($ EoS $)$ parameters $\omega_{\mathrm{sc}}(a)=$ $P_{\mathrm{sc}}(a) / \rho_{\mathrm{sc}}(a)$, in which $\omega_{\mathrm{sc}}(a)$ is a function of scale factor $a$. We can rewrite the above equation as

$$
\dot{\rho}_{\mathrm{sc}}+3 \frac{\dot{a}}{a} \rho_{\mathrm{sc}}\left[1+\omega_{\mathrm{sc}}(a)\right]=0 .
$$


Integrating the above equation, it is easy to get the solution for $\rho_{\mathrm{sc}}$

$$
\rho_{\mathrm{sc}}=\rho_{0}\left\{\frac{a_{0}^{3\left[\omega_{0}+1\right]}}{a^{3\left[\omega_{\mathrm{sc}}(a)+1\right]}}\right\} \exp \left[-3 \int_{a}^{a_{0}} d a \omega_{\mathrm{sc}}^{\prime}(a) \ln a\right],
$$

with $\rho_{0}, a_{0}, \omega_{0}$ denote today's energy density, scale factor and EoS parameter respectively. For $a_{0} \gg a_{\star}$, it's not necessary to consider the quantum effect of those present values (For conservation, we will define the subscript '0' denotes the present value of a physical quantity.). And the prime means the derivation with respect to the scale factor $a$.

In the above discussion, we defined the semiclassical energy density as $\rho_{\mathrm{sc}}=E_{\mathrm{m}} / a^{3}$, but this is not the only definition. Singh has showed us that there are two ways to obtain the semiclassical density [27]. One way is just like the definition we discussed above. The other one is to define a density operator $\hat{\rho}_{\mathrm{q}}=\widehat{H_{\mathrm{m}} / a^{3}}$ and then take their eigenvalues. The eigenvalues for $\hat{\rho}_{\mathrm{q}}$ can be obtained by considering $\hat{H}_{\mathrm{m}}$ and $\widehat{1 / a^{3}}[27]$

$$
\rho_{\mathrm{q}}=d_{j, l}(a) E_{m}(a, \phi)=D_{l}(q) a^{-3} E_{\mathrm{m}}(a, \phi)=D_{l}(q) \rho_{\mathrm{sc}},
$$

in which $d_{j, l}=D_{l}(q) a^{-3}$ is the eigenvalue for $\widehat{1 / a^{3}}$ for the large $j$. And $D_{l}$ is given as [27]

$$
\begin{aligned}
D_{l}(q)= & \left\{\frac { 2 7 | q | ^ { 1 - 2 l / 3 } } { 8 l } \left[\frac { 1 } { l + 3 } \left((q+1)^{2(l+3) / 3}\right.\right.\right. \\
& \left.-|q-1|^{2(l+3) / 3}\right)-\frac{2 q}{2 l+3}\left((q+1)^{2(l+3) / 3}\right. \\
& \left.\left.-\operatorname{sgn}(\mathrm{q}-1)|\mathrm{q}-1|^{2(\mathrm{l}+3) / 3}\right)\right\}^{\frac{3}{2-2 l}}
\end{aligned}
$$

where $q=a^{2} / a_{\star}^{2}$, and $l$ is the quantum ambiguity parameters with $0<l<1$ [26]. For $a \lesssim a_{\star}, D_{l} \lesssim 1$, this means $\rho_{\mathrm{sc}} \lesssim \rho_{\mathrm{q}}$.

Now, we turn to discuss the relationship between the semiclassical energy density and the classical one. The classical energy conservation is

$$
\dot{\rho}_{\mathrm{cl}}+3 H\left(\rho_{\mathrm{cl}}+P_{\mathrm{cl}}\right)=0
$$

Considering the varying EoS parameter $\omega_{\mathrm{cl}}(a)=\rho_{\mathrm{cl}} / P_{\mathrm{cl}}$, the above equation can be written as

$$
\dot{\rho}_{\mathrm{cl}}+3 H \rho_{\mathrm{cl}}\left[1+\omega_{\mathrm{cl}}(a)\right]=0
$$

It is easy to obtain the expression for $\rho_{\mathrm{cl}}$ from the above equation

$$
\rho_{\mathrm{cl}}=\rho_{0}\left\{\frac{a_{0}^{3\left[\omega_{0}+1\right]}}{a^{3\left[\omega_{\mathrm{cl}}(a)+1\right]}}\right\} \exp \left[-3 \int_{a}^{a_{0}} d a \omega_{\mathrm{cl}}^{\prime}(a) \ln a\right] .
$$

Considering the definition of $\rho_{\mathrm{q}}$, we can get the eigenvalues for $\widehat{\rho}_{\mathrm{q}}$ using the eigenvalues for $\widehat{1 / a^{3}}, d_{j, l}$, instead of $a^{-3}$ in Eq.(16). So, the semiclassical expression for $\rho_{\mathrm{q}}$ can be written as

$$
\begin{aligned}
\rho_{\mathrm{q}} & =\rho_{0}\left\{a_{0}^{3\left[\omega_{0}+1\right]} d_{j, l}^{\left[\omega_{\mathrm{cl}}(a)+1\right]}\right\} \exp \left[-3 \int_{a}^{a_{0}} d a \omega_{\mathrm{cl}}^{\prime}(a) \ln a\right] \\
& =D_{l}^{\omega_{\mathrm{cl}}(a)+1} \rho_{\mathrm{cl}}(a) .
\end{aligned}
$$

Remembering the relationship between $\rho_{\mathrm{q}}$ and $\rho_{\mathrm{sc}}$, which is described by Eq.(12), we can get the relationship between $\rho_{\text {sc }}$ and $\rho_{\mathrm{cl}}$

$$
\rho_{\mathrm{sc}}=D_{l}^{\omega_{\mathrm{cl}}(a)} \rho_{\mathrm{cl}}
$$

Now we want to get the relationship between $\omega_{\mathrm{sc}}(a)$ and $\omega_{\mathrm{cl}}(a)$. Differentiating $\ln \rho_{\mathrm{sc}}$ with respect to $\ln a$, one can obtain

$$
\frac{d \ln \rho_{\mathrm{sc}}}{d \ln a}=\ln D_{l} \frac{d \omega_{\mathrm{cl}}(a)}{d \ln a}+\omega_{\mathrm{cl}}(a) \frac{d \ln D_{l}}{d \ln a}+\frac{d \ln \rho_{\mathrm{cl}}(a)}{d \ln a} .
$$

Note that $\frac{d \ln \rho_{\mathrm{sc}}(a)}{d \ln a}=\frac{d \rho_{\mathrm{sc}}(a)}{H \rho_{\mathrm{sc}}(a) d t}=-3\left[1+\omega_{\mathrm{sc}}(a)\right]$, and $\frac{d \ln \rho_{\mathrm{cl}}(a)}{d \ln a}=\frac{d \rho_{\mathrm{cl}}(a)}{H \rho_{\mathrm{cl}}(a) d t}=-3\left[1+\omega_{\mathrm{cl}}(a)\right]$, it is easy to get

$$
\omega_{\mathrm{sc}}(a)=\omega_{\mathrm{cl}}(a)\left[1-\frac{1}{3} \ln D_{l} \frac{d \ln \left|\omega_{\mathrm{cl}}(a)\right|}{d \ln a}-\frac{1}{3} \frac{d \ln D_{l}}{d \ln a}\right] .
$$

Remembering that $\omega_{\mathrm{cl}}(a)$ may be a negative value for dark energy fluid, so we need choose the absolute value when we calculate its logarithm, but it dose not change the answer. Considering Eq.(20), it is easy to find that the effective EoS in loop quantum cosmology is possible " $1 "$ crossing. If $\omega_{\mathrm{cl}}=$ const., Eq.(20) will still hold, but the second term in the square brackets is zero. So the constant classical EoS will be a varying term in the effective region.

In this section, we get the equation of the effective EoS of dark energy with a varying EoS in loop quantum cosmology. Now, we can turn to consider the thermodynamical properties of dark energy in loop quantum cosmology scenario.

\section{THERMODYNAMICS PROPERTIES OF DARK ENERGY}

In this section, we will discuss the thermodynamics properties of dark energy in semiclassical regions. This section includes two subsections. In the first subsection, we will discuss the entropy-area relation of apparent horizon in semiclassical scenario. In the next subsection, we will show the temperature, chemical potential and entropy of dark energy fluid inner of the apparent horizon.

\section{A. Modified entropy-area relation}

According to the fact that the Einstein equation can be rewritten as the form $d E_{\mathrm{m}}=T d S+W_{\mathrm{m}} d V$ [28 30], 
with the total energy $E_{\mathrm{m}}=\rho V$ and the work density $W_{\mathrm{m}}=(\rho-p) / 2$, the authors of [16] showed that the entropy-area relationship of apparent horizon would be corrected by the modified Friedmann equation in loop quantum cosmology. In this subsection, we will discuss the modified entropy-area relation which is caused by the corrected Friedmann equation (6). Just as [16] stated, the temperature of apparent horizon is just determined by the spacetime metric, so it is convenient to assume the temperature of apparent horizon is $T_{\mathrm{A}}=1 /\left(2 \pi \tilde{r}_{\mathrm{A}}\right)$, with the radius of the apparent horizon $\tilde{r}_{\mathrm{A}}$. Noticed that the LQC is just correcting the Hamiltonian, not the Lagrangian. And the spacetime metric is described by the Lagrangian, so it is natural to believe that the definition of temperature of the apparent horizon in LQC is as same as in the classical one.

Our interested spacetime is the closed FriedmannRobertson-Walker (FRW) universe which is described by the metric

$$
\begin{aligned}
d s^{2} & =-d t^{2}+a^{2}\left(\frac{d r^{2}}{1-r^{2}}+r^{2} d \Omega_{2}^{2}\right) \\
& =h_{a b} d x^{a} d x^{b}+\tilde{r}^{2} d \Omega_{2}^{2},
\end{aligned}
$$

with $h_{a b}=\operatorname{diag}\left(-1, a^{2} /\left(1-r^{2}\right)\right), \tilde{r}=a(t) r$, and $k$ denotes the spatial curvature. If we define the Missner-Sharpe mass [24, 31, 32.

$$
h^{a b} \partial_{a} \tilde{r} \partial_{b} \tilde{r} \equiv f
$$

the apparent horizon is determined from $f=0$. So, we can get the radius of the apparent horizon

$$
\tilde{r}_{\mathrm{A}}=\frac{1}{\sqrt{H^{2}+1 / a^{2}}} .
$$

with the Hubble parameter $H$ described by Eq.(6).

In this paper, we consider the dark energy fluid is a perfect fluid satisfies $T_{\mu \nu}=\left(\rho_{\mathrm{sc}}+P_{\mathrm{sc}}\right) U_{\mu} U_{\nu}+P_{\mathrm{sc}} g_{\mu \nu}$, where $\rho_{\mathrm{sc}}$ and $P_{\mathrm{sc}}$ are the modified energy density and pressure in the semiclassical LQC, respectively. And the energy conservation law which is described by Eq.(10) still satisfied.

The Einstein equation is written as

$$
d E=A \Psi+W d V
$$

where $A=4 \pi \tilde{r}_{\mathrm{A}}^{2}$ is the area of the apparent horizon and $V=\frac{4 \pi}{3} \tilde{r}_{\mathrm{A}}^{3}$ is the volume of 3 -dimensional sphere. $E=M$ is the Misser-Sharp energy which is given by Eq.(22). $W$ is the work density and $\Psi$ is the energy-supply vector, are defined as

$$
\begin{array}{r}
W=-\frac{1}{2} T^{a b} h_{a b}, \\
\Psi_{a}=T_{a}^{b} \partial_{b} \tilde{r}+W \partial_{a} \tilde{r},
\end{array}
$$

where $T_{a b}$ is the projection of the $(3+1)$-dimensional energy-momentum tensor $T_{\mu \nu}$ of dark energy fluid in the FRW universe in the normal direction of 2-sphere 16.
Considering our interested metric (21), we can get the obvious expressions for $W, \Psi_{a}[16]$ :

$$
\begin{gathered}
W=\frac{1}{2}\left(\rho_{\mathrm{sc}}-P_{\mathrm{sc}}\right) \\
\Psi_{a}=-\frac{1}{2}\left(\rho_{\mathrm{sc}}+P_{\mathrm{sc}}\right) H \tilde{r} d t+\frac{1}{2}\left(\rho_{\mathrm{sc}}+P_{\mathrm{sc}}\right) a d r
\end{gathered}
$$

In those equations, we have considered the quantal correction.

The energy thrill through the apparent horizon at the interval time $d t$ is given as [16]

$$
\delta Q=-d E=-A \Psi=A\left(\rho_{\mathrm{sc}}+P_{\mathrm{sc}}\right) H \tilde{r}_{\mathrm{A}} d t
$$

with the area of the apparent horizon $A=4 \pi \tilde{r}_{\mathrm{A}}^{2}$. If the modified entropy of the apparent horizon is denoted as $S_{\mathrm{A}}^{\mathrm{sc}}$, considering $\delta Q=T d S_{\mathrm{A}}^{\mathrm{sc}}$ and Eq.(29), one can obtain

$$
\begin{aligned}
d S_{\mathrm{A}}^{\mathrm{sc}}= & 8 \pi^{2} \frac{1}{H^{3}}\left(\rho_{\mathrm{sc}}+P_{\mathrm{sc}}\right) d t \\
& =\frac{1}{8 \pi G}\left[\frac{2 \pi \dot{A}}{\mathcal{S}}+\frac{A^{2} H^{2}}{2} \frac{\dot{\mathcal{S}}}{\mathcal{S}^{2}}\right. \\
& \left.-\frac{1}{2} \frac{\dot{\mathcal{S}} A^{2}}{a^{2}}-\frac{1-\mathcal{S}^{2}}{a^{2}} \frac{H A^{2}}{\mathcal{S}}\right] d t
\end{aligned}
$$

in which we have used the temperature of the apparent horizon $T_{\mathrm{A}}=1 /\left(2 \pi \tilde{r}_{\mathrm{A}}\right)$ with the radius of the apparent horizon $\tilde{r}_{\mathrm{A}}=1 / \sqrt{H^{2}+1 / a^{2}}$ and Eq.(8). Expanding the above equation and integrating it, Eq.(30) can be written as

$$
\begin{aligned}
S_{\mathrm{A}}^{\mathrm{sc}}= & S_{\mathrm{Aa}}^{\mathrm{sc}}+\frac{A_{\mathrm{eff}}}{4 G}+\frac{1}{4 G} \int A_{\mathrm{eff}} d \ln \mathcal{S} \\
& +\frac{1}{8 \pi G} \int \frac{A_{\mathrm{eff}}^{2} H^{2}}{2} d \mathcal{S}-\frac{1}{16 \pi G} \int \frac{A_{\mathrm{eff}}^{2}}{a^{2} \mathcal{S}^{2}} d \mathcal{S} \\
& -\frac{1}{8 \pi G} \int \frac{\left(1-\mathcal{S}^{2}\right) \mathcal{S}}{a^{2}} H A_{\mathrm{eff}}^{2} d t,
\end{aligned}
$$

in which $A_{\text {eff }}=A / \mathcal{S}$ denotes the effective area of the apparent horizon in the semiclassical LQC. in which $S_{\mathrm{Aa}}^{\mathrm{sc}}$ is a constant and the value depends on the specific physics.

In a short conclusion, considering the Einstein equation (24) and assuming that the apparent horizon has Hawking temperature, we get the expression for the modified entropy-area relation in the semiclassical LQC. The semiclassical theory not only modify the entropy of apparent horizon, but also the area of it. And it is worth to notice that this modified entropy-area relation is not as same as the correction of black hole in loop quantum gravity 33.

$$
S_{\mathrm{bh}}=S_{\mathrm{BH}}+\sum_{n=0}^{\infty} C_{n} A_{H}^{-n}
$$

with some constants $C_{n}$. And it is different from the modified entropy-area relationship, which has the same 
form as Eq.(32), when one considers the effective LQC with holonomy corrections [16]. This difference is reasonable. Because we consider the inverse volume correction in the semiclassical regions, but the authors of [16] considered the holonomy correction. Notice that there are many differences between inverse volume correction and holonomy correction. For example, the inverse volume correction modifies the Klein-Gorden equation [34], but holonomy correction does not 35]; and the semiclassical energy density and effective pressure which caused by the inverse volume correction are different from the ones caused by the holonomy correction [36].

\section{B. The thermodynamical variables of dark energy fluid}

In this section, we will discuss the thermodynamics properties of the dark energy in the inner side of the apparent horizon. Some authors advised that the temperature of dark energy fluid has the same temperature of the apparent horizon which the temperature is described by Hawking temperature, but this may be get a ill-defined entropy when $\omega_{\mathrm{cl}}<-1[24]$. And other authors find the dark energy either have negative entropy or temperature in the classical regions [21 23]. In this subsection, we will consider the temperature and entropy of the dark energy in the inner side of the apparent horizon. And we will assume the dark energy is a ideal fluid and with the chemical potential. This subsection, we will follow the method of [20] to discuss the temperature and entropy of the dark energy in semiclassical LQC scenario.

The entropy fluid and particle fluid are described by $S_{\mathrm{sc}}^{\mu}=s_{\mathrm{sc}} u^{\mu}$ and $N^{\mu}=n u^{\mu}$, with the particle number density $n$ and the entropy density $s_{\mathrm{sc}}$, respectively. Considering they satisfy $S_{; \mu}^{\mu}=0, N_{; \mu}^{\mu}=0$, it is easy to obtain

$$
\begin{array}{r}
\dot{s}_{\mathrm{sc}}+3 H s_{\mathrm{sc}}=0, \\
\dot{n}+3 H n=0 .
\end{array}
$$

The solutions for above equations are

$$
n(a)=n_{0}\left(\frac{a}{a_{0}}\right)^{3}, \quad s_{\mathrm{sc}}(a)=s_{0}\left(\frac{a}{a_{0}}\right)^{3} .
$$

Now we consider the thermodynamics. We assume the thermodynamic properties is described by the particle number density $n$ and the temperature $T_{\mathrm{sc}}$. We have assumed the quantum effect will modify the temperature. This is reasonable. If assuming that the modified energy density and the temperature is still satisfy the Stenfan-Boltzmann law $\rho_{\mathrm{sc}} \propto f\left(T_{\mathrm{sc}}\right)$, the temperature $T_{\text {sc }}$ should be dependent on $a$, for the energy density is the function of the scale factor $a$, and then the temperature should be modified by the semiclassical quantum effect. We will see that the Stenfan-Boltzmann law still is tenable in our model later on. We consider the Gibbs law, $T_{\mathrm{sc}}\left(\frac{\partial P_{\mathrm{sc}}}{\partial T_{\mathrm{sc}}}\right)_{n}=\rho_{\mathrm{sc}}+P_{\mathrm{sc}}-n\left[\frac{\partial \rho_{\mathrm{sc}}(a)}{\partial n}\right]_{T_{\mathrm{sc}}}$, then the prefect fluid can be described by

$T_{\mathrm{sc}}(a)\left[\frac{\partial \omega_{\mathrm{sc}}(a) \rho_{\mathrm{sc}}}{\partial T_{\mathrm{sc}}(a)}\right]_{n}=\left(\omega_{\mathrm{sc}}(a)+1\right) \rho_{\mathrm{sc}}-n\left[\frac{\partial \rho_{\mathrm{sc}}(a)}{\partial n}\right]_{T_{\mathrm{sc}}}$.

Considering $\dot{\rho}_{\mathrm{sc}}(a)=\dot{n}\left(\frac{\partial \rho_{\mathrm{sc}}(a)}{\partial n}\right)_{T_{\mathrm{sc}}(a)}+\dot{T}_{\mathrm{sc}}(a)\left(\frac{\partial \rho_{\mathrm{sc}}(a)}{\partial T_{\mathrm{sc}}(a)}\right)_{n}$ and Eqs.(10) and (36), we can get the evolution of modified temperature with respect to the semiclassical EoS parameters

$$
\left[\frac{3}{a} T_{\mathrm{sc}}(a) \omega_{\mathrm{sc}}(a)+T_{\mathrm{sc}}^{\prime}(a)\right]\left(\omega_{\mathrm{sc}}(a)+1\right)=T_{\mathrm{sc}}(a) \omega_{\mathrm{sc}}^{\prime}(a) .
$$

Just as the mention in Sec II the modified EoS parameters $\omega_{\mathrm{sc}}(a)$ are possible -1 crossing. It is obvious that the modified temperature which is described by above equation is zero when $\omega_{\mathrm{sc}}(a)=-1$ for $\omega_{\mathrm{sc}}^{\prime}(a) \neq 0$. Integrating the above equation when $\omega_{\mathrm{sc}}(a) \neq-1$, we can get the expression for $T_{\mathrm{sc}}(a)$

$$
\begin{aligned}
T_{\mathrm{sc}}(a)= & T_{0}\left(\frac{\omega_{\mathrm{sc}}(a)+1}{\omega_{0}+1}\right)\left(\frac{1}{a^{3 \omega_{\mathrm{sc}}(a)}}\right) \\
& \times \exp \left[-3 \int_{a}^{1} d a \omega_{\mathrm{sc}}^{\prime}(a) \ln a\right],
\end{aligned}
$$

with the present temperature $T_{0}$. We have considered that the present scale factor $a_{0}=1$. There are four points should be noticed. First, if $\omega_{0}=-1$ and $T_{0} \neq 0$, $T_{\mathrm{sc}}(a)$ will be divergent. But if we consider $\omega_{\mathrm{sc}}(a)$ describes today's dark energy, $\omega_{\mathrm{sc}}(a)$ should not be modified by the quantum effect for $a_{0} \gg a_{\star}$ and is substituted by $\omega_{\mathrm{cl}}(a)$. In this case, Eq.(37) describes the temperature for the dark energy in the classical regions. The temperature of dark energy with $\omega\left(a_{0}\right)=-1$ should be zero, for $\left.\omega_{\mathrm{cl}}^{\prime}(a)\right|_{a=a_{0}} \neq 0$ in Eq.(37). So, $T_{0}=0$ when $\omega_{0}=-1$ if the EoS $\omega_{\mathrm{cl}}$ is a function of the scale factor. Second, the temperature is zero when $\omega_{\mathrm{sc}}(a)=-1$. But this "- 1 " EoS parameter is caused by the effective behavior. Third, for $T_{0}$ and $\omega_{0}+1$ have the same sign, if $\omega_{\mathrm{sc}}(a)>-1$ the temperature is positive, the temperature is negative when $\omega_{\mathrm{sc}}(a)<-1$, and $T_{\mathrm{sc}}(a)=0$ when $\omega_{\mathrm{sc}}(a)=-1$, this is as same as the behavior of temperature in the classical regions [20]. But notice that $\omega_{\mathrm{sc}}$ are modified EoS parameters and includes the quantum geometry effect. Fourth, the scale factor is in the regions $a_{i}<a<a_{\star}$, so $\frac{1}{a}$ in Eq.(38) will not divergent, this is different from [20] in which the authors considered the thermodynamical properties of dark energy in the classical regions.

Now we have the expressions for modified temperature and modified energy density. It is easy to obtain the 
generalized Stefan-Boltzmann law

$$
\begin{aligned}
& \rho_{\mathrm{sc}}(a)= \rho_{0}\left[\frac{T_{\mathrm{sc}}(a)}{T_{0}} \frac{\omega_{0}+1}{\omega_{\mathrm{sc}}(a)+1}\right]^{\frac{\omega_{\mathrm{sc}}(a)+1}{\omega_{\mathrm{sc}}(a)}} \\
& \times \exp \left[\frac{3}{\omega_{\mathrm{sc}}(a)} \int_{a}^{1} d a \omega_{\mathrm{sc}}^{\prime}(a) \ln a\right], \\
& \rho_{\mathrm{sc}}(a)= \rho_{0}\left[\frac{T_{\mathrm{sc}}(a)}{T_{0}} \frac{\omega_{0}+1}{\omega_{\mathrm{sc}}(a)+1}\right]\left(\frac{1}{a}\right)^{3}, \\
& \text { for } \quad \omega_{\mathrm{sc}}(a)=0 .
\end{aligned}
$$

It is worth to notice that the above equations are still valid for $\omega_{\mathrm{sc}}(a)=-1$, for $T_{\mathrm{sc}}(a)=0$ in this moment.

Now we turn to consider the chemical potential and entropy. Consider the Euler's relation of $T_{\mathrm{sc}}(a) s_{\mathrm{sc}}(a)=$ $\left[1+\omega_{\mathrm{sc}}(a)\right] \rho_{\mathrm{sc}}(a)-\mu_{\mathrm{sc}}(a) n$, with the chemical potential $\mu_{\mathrm{sc}}(a)$, the chemical potential can be given as [20]

$$
\mu_{\mathrm{sc}}(a)=\frac{1}{n}\left[\left(1+\omega_{\mathrm{sc}}(a)\right) \rho_{\mathrm{sc}}(a)-T_{\mathrm{sc}}(a) s_{\mathrm{sc}}(a)\right] .
$$

Using the Gibbs law (36) and the modified temperature (38), it is easy to get the modified chemical potential

$$
\begin{aligned}
\mu_{\mathrm{sc}}(a)= & \mu_{0}\left[\frac{\omega_{\mathrm{sc}}(a)+1}{\omega_{0}+1}\right]\left[\frac{1}{a^{3 \omega_{\mathrm{sc}}(a)}}\right] \\
& \times \exp \left[-3 \int_{a}^{1} d a \omega_{\mathrm{sc}}^{\prime}(a) \ln a\right],
\end{aligned}
$$

with chemical potential at present $\mu_{0}=\frac{1}{n_{0}}\left[\rho_{0}\left(\omega_{0}+1\right)-\right.$ $\left.T_{0} s_{0}\right]$. As before, the above equation is well-defined when $\omega_{0}=-1$, for $T_{0}=0$. But the sign of $\mu_{\mathrm{sc}}(a)$ is independent on $\omega_{\mathrm{sc}}(a)$, for the sign of $\mu_{0}$ can be general. And the chemical potential can be wrote as a function of $T_{\mathrm{sc}}(a)$,

$$
\mu_{\mathrm{sc}}(a)=\mu_{0} \frac{T_{\mathrm{sc}}(a)}{T_{0}} .
$$

It is easy to get the entropy of the dark energy fluid by considering the Eq.(40). Remembering $S(a)=$ $s(a) V(a)=s(a) a^{3}$, and using Eqs.(35), (39) and (42), we can get the expression for entropy.

$$
\begin{aligned}
S_{\mathrm{sc}}(a)= & s_{0} V(a)\left[\frac{T_{\mathrm{sc}}(a)}{T_{0}} \frac{\omega_{0}+1}{\omega_{\mathrm{sc}}(a)+1}\right]^{\frac{1}{\omega_{\mathrm{sc}}(a)}} \\
& \times \exp \left[\frac{3}{\omega_{\mathrm{sc}}(a)} \int_{a}^{1} d a \omega_{\mathrm{sc}}^{\prime}(a) \ln a\right],
\end{aligned}
$$

in which we have considered the present scale factor $a_{0}=$ 1. When $\omega_{\mathrm{sc}}(a)=0$, combining Eq.(39) and Eq.(42), we find it is a trivial result. Note that, above equation is regular at $\omega_{\mathrm{sc}}(a)=-1$, because if $\omega_{\mathrm{sc}}(a)=-1, T_{\mathrm{sc}}=0$. And if $\omega_{0}=-1$, then $T_{0}=0$, so the above equation is still well-defined. It is easy to get $s_{0} V_{0}=s_{\mathrm{sc}}(a) V(a)$.

In a short conclusion, in this subsection, we has discussed the thermodynamics properties of dark energy in the inside of apparent horizon, and get the expressions for temperature, chemical potential and entropy. We find that those variables are corrected due to the semiclassical quantum effect, and those corrections are connected with the modified EoS parameters. Our method is following [20], in which the temperature is regular for the vacuum when they discuss the thermodynamics properties in the classical regions but the temperature will be divergent at $a=0$. But due to the modification of semiclassical quantum effect, $a=0$ doesn't lie in the regions we interested, so the expression for temperature always holds for a varying classical EoS $\omega_{\mathrm{cl}}$. The expressions for chemical potential and entropy are obtain in this subsection.

\section{CONCLUSION AND DISCUSSION}

In this paper, we have discussed the thermodynamics properties of the dark energy in the semiclassical LQC scenario. The common feature of the all models of dark energy is the EoS parameters $\omega$ of dark energy fluid is included by the field evolution. So, at the first, we consider the correction of EoS parameters in the regions we interested. We find that the corrected EoS parameters depends on the changing rate of inverse volume factor $D_{l}$ and classical EoS parameters $\omega_{\mathrm{cl}}(a)$.

We discussed the area-entropy relation of the apparent horizon of universe which is full of dark energy fluid. We found that the effective LQC not only modify the entropy of apparent horizon, but also the area of it. And the modified area-entropy relation is different from the one considered by [16], in which the authors considered the holonomy correction. And then, we discussed the thermodynamical properties of dark energy fluid in the inner side of apparent horizon. The expressions for temperature, chemical potential and entropy are obtained. We find that, if the classical EoS parameter is a function of the scale factor, the thermodynamical variables are functions of the modified EoS parameters and well-defined when $\omega\left(a_{0}\right)=-1$. The sign of temperature is dependent on the modified EoS $\omega_{\mathrm{sc}}$, when $\omega_{\mathrm{sc}}>-1, T_{\mathrm{sc}}>0, T_{\mathrm{sc}}<0$ when $\omega_{\mathrm{sc}}<-1$, and $T_{\mathrm{sc}}=0$ if $\omega_{\mathrm{sc}}=-1$, but the sign of the modified temperature is independent on the sign of potential, which is determined by the sign of $\mu_{0}$. This is as same as the discussion in the classical region [20]. $T_{\mathrm{sc}}(a)=S_{\mathrm{sc}}(a)=0$ when $\omega_{\mathrm{sc}}(a)=-1$, this is like the behavior of massless degenerate Fermi gas [37, 38]. Just as we discussed above, when $\omega_{\mathrm{sc}}<-1$, the temperature is negative. But this negative temperature is independent on the sign of potential, for the sign of potential is just dependent on $\mu_{0}$. The negative temperature can only be explain in the quantum framework. The quantum field theory for the field with EoS parameters $\omega_{\mathrm{cl}}<-1$ is still an open question. In the semiclassical regions, the EoS parameters should be modified by the quantum geometry effect, so the negative temperature in the semiclassical regions partial is cased by the quantum geometry effect.

In this paper, we just consider the thermodynamics properties of dark energy fluid, it is easy to verify that 
our method can apply to the other kinds of matter, e.g., stiff matter, radiation and so on, for the EoS of this matter will be modified in the effective region in loop quantum cosmology, just as Singh shown [27]. But this model can not describe the thermodynamics properties of cosmological constant with the constant EoS -1. Although the EoS of cosmological constant will be modified in the effective region, just as Eq.(20) described. The temperature of cosmological constant today is still unclear, so the term $T_{0} /\left(\omega_{0}+1\right)$ in Eq.(38) will be divergent for $\omega_{\mathrm{sc}}$ does not always equal to -1 .

\section{Acknowledgments}

The work was supported by the National Natural Science of China (No. 10875012) and the Scientific Research Foundation of Beijing Normal University.
[1] A. Melchiorri, L. Mersini, C. J. Odman and M. Trodden, Phys. Rev. D 68, 043509(2003).

[2] E. J. Copeland, M. Sami and S. Tsujikawa, Int.J.Mod.Phys.D 15,1753(2006); R. Rakhi and K. Indulekha, arXiv:0911.2601; A. Kesavan, Dark energy, arXiv:0908.2852.

[3] Z. K. Guo, N. Ohta and Y. Z. Zhang, Phys. Rev. D 72, 023504(2005);

[4] P. Singh, M. Sami and N. Dadhich, Phys. Rev. D 68, 023522(2003); V. B. Johri, Phys. Rev. D 70, 041303(R)(2004); L. P. Chimento and R. Lazkoz, hep-th/0405518

[5] B. Fang, X. L. and X. M. Zhang, Phys. Lett. B 607, 35,(2005); W. Zhao and Y. Zhang, Phys. Rev. D 73,123509(2006); Y. P. Zhang, Z. L. Yi, T.J. Zhang and W. B. Liu, Phys. Rev. D 77, 023502 (2008).

[6] Q. Shafi, A. Sil and Siew-Phang Ng, Phys. Lett. B 620, 105(2005); A. de la Macorra, F. Briscese, AIP Conf. Proc. 1116, 179(2009); N. Bose, A. S. Majumdar,Phys. Rev. D 79, 103517(2009); Philippe Brax, Carsten van de Bruck, L. M. H. Hall, J. M. Weller,Phys. Rev. D 79, 103508(2009).

[7] Z. K. Guo, Y. S. Piao, and Y. Z. Zhang, Phys. Lett. B 594, 247(2004); J. G. Hao and X. Z. Li, Phys. Rev. D 68, 083514(2003).

[8] M. D. Maia, E. M. Monte, J. M. F. Maia, and J.S. Alcaniz, Class. Quant. Grav. 22, 1623(2005); M. D. Maia, A. J. S. Capistrano, J. S. Alcaniz, and E. M. Monte, arXiv:0905.4259.

[9] M. Sami, Mod. Phys. Lett. A 19, 1509 (2004); R. R. Caldwell, M. Kamionkowshi, and N. N. Weinberg, Phys. Rev. Lett. 91, 071301(2003).

[10] S. Nojiri, S. D. Odintsov, and S. Tsujikawa, Phys. Rev. D 71, 063004(2005); S. Carneiro, R. Tavakol, Gen. Rel. Grav. 41, 2287(2009).

[11] P. F. Gonzalez-Diaz, and C. L. Siguenza, Nucl. Phys. B 697, 363(2004); S. H. Pereira and J. A. S. Lima, Phys. Lett. B 669, 266(2008).

[12] J.S. Alcaniz, D. Jain, A. Dev, Phys. Rev. D 66, 067301(2002); M. Sami, P. Singh, S. Tsujikawa, Phys. Rev. D 74, 043514(2006); S.B.Chen, B. Wang, J.L. Jing, Phys. Rev. D78 123503(2008); X. Y. Fu, H. W. Yu,and P. X. Wu, Phys. Rev. D78, 063001(2008); A. de la Macorra, F. Briscese, AIP Conf. Proc. 1116, 179(2009); E. S. Corchero, AIP Conf. Proc.1122, 229(2009); Y.P. Zhang, Z.L. Yi, T.J. Zhang, and W.B. Liu, Phys. Rev. D

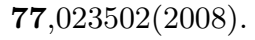

[13] G. W. Gibbons and S. W. Hawking, Phys. Rev. D 15, 2738(1977); M. D. Pollock and T. P. Singh, Class. Quant. Grav. 6, 901(1989); A. V. Frolov and L. Kofman, JCAP 0305,009(2003).

[14] B. Wang, Y. G. Gong and E. Abdalla, Phys. Rev. D 74, 083520 (2006).

[15] L. F. Li and J. Y. Zhu, Advances in High Energy Physics, 2009, 905705(2009)

[16] R. G. Cai, L.M . Cao and Y. P. Hu, JHEP 08, 090(2008).

[17] M. Bojowald, Living Rev. Rel. 11, 4(2008).

[18] A. Ashtekar, J. Phys .Conf. Ser. 189, 012003(2009); A. Ashtekar, Gen. Rel. Grav. 41, 707(2009).

[19] A. Ashtekar, M. Bojowald and J. Lewandowski, Adv. Theor. Math. Phys. 7, 233(2003).

[20] E. N. Saridakrics, P. F. Gonzalez-Diza and C. L. Siguenza, Class. Quantum Grav. 26, 165003(2009).

[21] P. F. Gonzalez-Diaz and C. L. Siguenza, Nucl. Phys. B 697, 363(2004).

[22] Y. S. Myung, Phys. Lett. B 671, 216(2009).

[23] J. A. S. Lima, J. S. Alcaniz, Phys. Lett. B 600, 191(2004); J. A. S. Lima, S. H. Pereira, Phys.Rev.D 78, 083504(2008).

[24] Y. G. Gong, B. Wang and A. Z. Wang, JCAP 01, 024(2007); Y. G. Gong, B. Wang and A. Z. Wang, Phys. Rev. D 75, 123516(2007)

[25] J. Magueijo and P. Singh, Phys. Rev. D76, 023510(2007).

[26] K. Vandersloot, Phys. Rev. D71, 103506(2005).

[27] P. Singh, Class. Quantum Grav. 22, 4203(2005).

[28] A. V. Frolov and L. Kofman, JCAP 05, 009(2003).

[29] U. H. Danielsson, Phys. Rev. D 71, 023516(2005).

[30] R. Bousso,Phys. Rev. D 71, 064024(2005).

[31] E. Poisson and W. Israel, Phys. Rev. D 41, 1796(1990).

[32] C. M. Misner and D. H. Sharpe, Phys. Rev.136, B571(1964).

[33] R. K. Kaul and P. Majumdar, Phys. Rev. Lett. 84, $5255(2000)$.

[34] S. Tsujikawa, P. Singh and R. Maartens, Class. Quan. Grav. 21, 5767, (2004).

[35] M. Artymowski, Z. Lalak and L. Szulc, JCAP 01, 04(2009).

[36] E.J. Copeland, D. J. Mulryne, N. J. Nunes and M. Shaeri, Phys. Rev. D 77, 023510(2008).

[37] N. Bilic, Fortschr. Phys. 56, 363(2008).

[38] P. B. Pal, An Introductory Course of Statistical Mechanics (Alpha Science, Oxford, U.K.). 